\title{
Statistically stable ultrasonic imaging in random media
}

\author{
James G. Berryman* $\quad$ Liliana Borcea ${ }^{\dagger} \quad$ George C. Papanicolaou $^{\ddagger} \quad$ Chrysoula Tsogka $^{\S}$
}

\begin{abstract}
Analysis of array data from acoustic scattering in a random medium with a small number of isolated targets is performed in order to image and, thereby, localize the spatial position of each target. Because the host medium has random fluctuations in wave speed, the background medium is itself a source of scattered energy. We assume, however, that the targets are sufficiently larger and/or more reflective than the background fluctuations so that a clear distinction can be made between targets and background scatterers. In our numerical simulations we use nonreflective boundary conditions so as to isolate the effects of the host randomness from those of the spatial boundaries, which can then be treated in a separate analysis. We show that the key to successful imaging is finding statistically stable functionals of the data whose extreme values provide estimates of scatterer locations. The best ones are related to the eigenfunctions and eigenvalues of the array response matrix, just as one might expect from prior work on array data processing in complex scattering media having homogeneous backgrounds. The specific imaging functionals studied include matched-field processing and linear subspace methods, such as MUSIC (MUltiple SIgnal Classification). But statistical stability is not characteristic of the frequency domain, which is often the province of these methods. By transforming back into the time domain after first diagonalizing the array data in the frequency domain, we can take advantage of both the time-domain stability and the frequency-domain orthogonality of the relevant eigenfunctions.
\end{abstract}

PACS numbers: 43.60.Pt,43.60.Gk,43.30.Vh,43.30.Wi

\section{INTRODUCTION}

Imaging in ultrasonics is closely related to recent studies of time-reversal acoustics that have been experiencing a very rapid growth in interest and research activity since the early work of (Fink et al., 1989), (Jackson and Dowling, 1991), and (Prada et al., 1991). In particular, a series of review articles (Fink, 1997; Fink, 1999; Fink et al., 2000; Fink and Prada, 2001; Fink, 2001; ter Haar, 2001) has appeared recently that, when taken altogether, largely summarizes the current state-of-the-art.

It is important to distinguish physical time-reversal and re-emission of the acoustic signals by the array, so as to produce optimal focusing on a target, from synthetic time-reversal processing in which the acoustic array data are used to estimate the locations of the scatterers. The work of (Prada and Fink, 1994) and (Prada et al., 1996) on the D.O.R.T. method (French acronym for a diagonalization of the array response matrix) has clarified the connection between individual scattering objects and the eigenfunctions of the time-reversal

\footnotetext{
${ }^{*}$ University of California, Lawrence Livermore National Laboratory, P.O.Box 808 L-200, Livermore, CA 94551-9900, USA

${ }^{\dagger}$ Computational and Applied Mathematics, Rice University, Houston, TX 77005-1892, USA

${ }^{\ddagger}$ Department of Mathematics, Stanford University, Stanford, CA 94305, USA

${ }^{\S}$ CNRS/LMA, 31 Chemin Joseph Aiguier, 13402 Marseille Cedex 20, FRANCE
} 
operator. Scatterers can be associated directly with eigenfunctions except when they are not well separated or when they are placed in some special, symmetric positions relative to the array (Devaney, 2002). Such degeneracies are, however, not important in random media.

After decomposing the array response matrix using eigenfunctions (this is normally done in the frequency domain), two alternatives are available for us to pursue. Either we can use the eigenfunctions to refocus acoustic energy back onto the scattering target (for purposes either of communication or medical ultrasound treatments - see, for example, (Thomas and Fink, 1996; ter Haar, 2001)), or we can try to make use of them to localize or form an image of the scatterers' spatial distribution. Both of these applications are relatively straightforward if the background medium is itself homogeneous (Mast et al., 1997). But, if the background medium is heterogeneous (i.e., the acoustic wave speed varies randomly with position), then the difficulties rapidly mount for both applications even if there are no additional complications. Such complications might, for example, include (1) near/close boundaries as will commonly occur in waveguides (Tanter et al., 1998; Mordant et al., 1999) (including the ocean bottom and surface for ocean acoustics (Kuperman et al., 1998)), (2) drift of the actual acoustic medium itself or just its physical properties due to currents or temperature fluctuations (Floch et al., 1999), (3) periodic changes such as expansion and contraction due to the breathing of a patient while being diagnosed or treated with ultrasound.

Our focus in this paper will be to ignore any additional complications, and concentrate instead on what difficulties are introduced just by the spatial heterogeneity of the acoustic medium, and what can be done with acoustic array data to achieve reliable images or maps of any significant scatterers present in such media. One companion paper (Blomgren et al., 2002) addresses the issues of refocusing sound at a target in the presence of a heterogeneous acoustic background with significant multipathing (multiple scattering), and some of the results on imaging presented here are an outgrowth of another companion paper (Borcea et al., 2002) on acoustic imaging in random media.

It is beyond our current scope to review the literature on acoustics in random media, but we will mention a few relevant references. Early work by (Keller, 1962; Keller, 1964) and (Karal Jr. and Keller, 1964; Keller and Karal Jr., 1966) has shown that the averaged Green's function in random media is a well-behaved quantity and how the effective wave speed and apparent attenuation due to multiple scattering both depend on the statistics of the fluctuating acoustic wave speed. These and many other topics are presented in (Ishimaru, 1978). Wave front stabilization in complex and especially in layered media has been observed (O'Doherty and Anstey, 1971) and studied extensively in (Richards and Menke, 1983; Burridge et al., 1986; Burridge et al., 1987; Burridge et al., 1988; Burridge et al., 1989; Clouet and Fouque, 1997; Solna and Papanicolaou, 2000).

There have been many methods of estimating target location using acoustic array data. Some of the most popular ones in recent years have continued to be matched-field processing (Bucker, 1976; Baggeroer et al., 1988; Baggeroer et al., 1993; Krolik, 1992; Jensen et al., 1994), MUSIC (MUltiple SIgnal Classification) (Schmidt, 1979; Schmidt, 1986; Devaney, 2002), and other linear subspace methods (Johnson, 1982; Johnson and DeGraaf, 1982; Cheney, 2001). We will be discussing necessary modifications of these methods here, since the randomness we consider has a different character than that usually envisioned in these traditional analyses of acoustic array data, because it comes from multipathing that is generated by the random medium.

Typical array processing methods assume that a source is located at a great distance from the array, while the propagating medium is homogeneous, so that from the point of view of the array the target looks like a point source. The incident sound arriving at the array takes the form of a plane-wave having no measurable curvature across the array aperture and, furthermore, has no measurable amplitude variation across that aperture. Array noise has usually been treated as due either to diffuse sources of white noise coming simultaneously from all directions, or to isolated "noise" sources having the same types of source characteristics as the targets of interest. Because the applications of interest have often involved passive 
bearing detection of active sources, most of the methods and their minor variations considered here were first introduced for such passive data collection and processing problems. But, for time-reversal methods, it quickly becomes clear that virtually the same methods - except for some obvious minor technical differences - should be considered for both active and passive arrays.

Time-reversal processing of the array response data is most naturally based from the outset on the Singular Value Decomposition (SVD). This approach is consistent with the concept of real-space time-reversal — involving an iterative procedure that amounts to using the power method for finding the singular vector of the data matrix having the largest singular value. When the full response/transfer matrix has been measured for a multistatic active array, the resulting data matrix can be analyzed directly by SVD to determine not only the singular vector having the largest singular value, but all singular vectors and singular values simultaneously. There remain some issues about signal-to-noise ratios suggesting that the physical iterative approach to finding these same singular vectors can be preferable to the purely computational alternative both in acoustics (Sabra et al., 2002) and also in other inverse problems (Cheney et al., 1999). But it is also important to recognize that the SVD of the transfer matrix does not require specialized hardware (any acoustic array can be used to do this), whereas the physical time-reversal approach for ultrasound does require very specialized and often quite expensive hardware. Thus, SVD may have a distinct advantage in some time-reversal imaging applications.

For acoustical imaging purposes, we do not need to propagate the actual time-reversed signal back into the physical random medium, whereas for either communication or target retrofocusing applications, such real-space backpropagation is always required. From this point of view, time-reversal imaging can also be seen as a relatively inexpensive process. The drawback is that imaging is always done using a fictitious medium for the simulated backpropagation that produces these images since the real medium is not known. Its large-scale features could be estimated from other information, such as geological data obtained by seismic methods. For example, migration methods (Claerbout, 1976; Aki and Richards, 1980; Bleistein et al., 2001) can be used, where very large arrays - much larger than those we contemplate using here are required. However, the small-scale random inhomogeneities are not known and cannot be effectively estimated, so the simplest thing to do is ignore them when imaging.

The following results are based in part on another analytical and computational study of time-reversal in random media by the present authors (Borcea et al., 2002). In the first section, we briefly present the problem to be studied and then elucidate the notation to be used in the following sections. Then, we present a series of examples - in order to compare and contrast the results. Section II focuses on the standard matched-field and MUSIC objective functionals in the frequency domain. These methods do not provide statistically stable results and, therefore, are not useful for imaging in media with randomly fluctuating acoustic wave speed and strong multipathing. Section III then shows how these same objective functionals may be transformed into the time domain in order to produce statistically stable and, therefore, useful images that localize the target cross-range (or bearing) in a satisfactory manner. Section IV then goes further to show how range information may be obtained from the time-domain arrival data after careful processing and subsequent averaging of multiple copies of the pertinent singular vectors contained in the multistatic array data. Synthetic Aperture Imaging (SAI) is also used to provide another source of comparison to a familiar data processing scheme. Our conclusions are summarized in Section V.

\section{Problem Statement and Notation}

An array has $N$ transducers located at spatial positions $\mathbf{x}_{p}$, for $p=1, \ldots, N$. When used in active mode, the array probes the unknown acoustic medium containing $M$ small scatterers by emitting pulses and recording the time traces of the back-scattered echos. We call the resulting data set the multistatic array response (or transfer) matrix $P(t)=\left(P_{p q}(t)\right)$, where $p$ and $q$ both range over all the array elements. For our simulations, 
we consider a linear array where two adjacent point transducers are a distance $\lambda / 2$ apart, with $\lambda$ being the carrier (central) wavelength of the probing pulses. Such an arrangement ensures that the collection of transducers behaves like an array having aperture $a=(N-1) \lambda / 2$ and not like separate entities, while keeping the interference among the transducers at a minimum (Steinberg, 1983). Our goal is to detect and then localize all $M$ of the targets in the random medium, if possible.

The array response matrix $\widehat{P}(\omega)$ in the frequency domain is, in our linear acoustics simulations, symmetric but not Hermitian. The singular value decomposition (SVD) of the response matrix is given explicitly by

$$
\widehat{P}(\omega)=\widehat{U}(\omega) \Sigma(\omega) \widehat{V}^{H}(\omega) .
$$

The eigenvectors of $\widehat{P}(\omega) \widehat{P}^{H}(\omega)$ having unit norm, denoted by $\widehat{\mathbf{U}}_{r}(\omega)$, for $r=1, \ldots, N$, are the columns of matrix $\widehat{U}(\omega)$. The eigenvalues of $\widehat{P}(\omega) \widehat{P}^{H}(\omega)$ are $\sigma_{r}^{2}(\omega)$, with $\sigma_{r}(\omega)$ being the singular values of $\widehat{P}(\omega)$ that form the diagonal matrix $\Sigma(\omega)$. The significant singular vectors $\widehat{\mathbf{U}}_{r}(\omega)$ [i.e., those in the range of $\widehat{P}(\omega)$ ] corresponding to singular values $\sigma_{r}(\omega)>0$ for $1 \leq r \leq M$, where $M$ is either the number of targets, or the size of the array $(N)$ - whichever is smaller. For definiteness, we usually assume that the number of targets is smaller than the array size $N$, so that $M$ is in fact the number of distinguishable targets. In our setup, the left singular vectors $\widehat{\mathbf{U}}_{r}(\omega)$ are the complex conjugates of the right singular vectors $\widehat{\mathbf{V}}_{r}(\omega)$, for $r=1, \ldots, N$. Most physical arrays, however, are not constructed with isotropic point transducers and, furthermore, the amplitude response (especially in transmission mode) does not have to be linear (with strong deviations from linearity generally occurring at both high and very low amplitudes), so measured response matrices are not necessarily symmetric. We assume symmetry here for simplicity, and this is fully consistent with our linear acoustics simulations. All of our analysis nevertheless carries over to the non-symmetric case.

We denote by $\widehat{\mathbf{g}}_{0}\left(\mathbf{y}^{s}, \omega\right)$ the vector observed at the array for a source located at $\mathbf{y}^{s}$ in a deterministic medium (i.e., the medium with the wave speed $c_{0}$ given by the averaged velocity of the random medium). In our simulations, $c_{0}$ is constant but, in general, it could vary in space - assuming prior knowledge of the environment. Then, $\widehat{\mathbf{g}}_{0}(\mathbf{y}, \omega)$ is given by

$$
\widehat{\mathbf{g}}_{0}\left(\mathbf{y}^{s}, \omega\right)=\left(\begin{array}{c}
\widehat{G}_{0}\left(\mathbf{y}^{s}, \mathbf{x}_{1}, \omega\right) \\
\widehat{G}_{0}\left(\mathbf{y}^{s}, \mathbf{x}_{2}, \omega\right) \\
\vdots \\
\widehat{G}_{0}\left(\mathbf{y}^{s}, \mathbf{x}_{N}, \omega\right)
\end{array}\right)
$$

where $\widehat{G}_{0}\left(\mathbf{y}^{s}, \mathbf{x}_{j}, \omega\right)$ is the deterministic two-point Green's function, and $\mathbf{x}_{j}$ is the location of the $j$-th array element.

We also define the projection $\mathcal{P}_{N} \widehat{\mathbf{g}}_{0}(\mathbf{y}, \omega)$ of $\widehat{\mathbf{g}}_{0}\left(\mathbf{y}^{s}, \omega\right)$ onto the null-space of $\widehat{P} \widehat{P}^{H}(\omega)$ by

$$
\mathcal{P}_{N} \widehat{\mathbf{g}}_{0}\left(\mathbf{y}^{s}, \omega\right)=\widehat{\mathbf{g}}_{o}\left(\mathbf{y}^{s}, \omega\right)-\sum_{r=1}^{M}\left[\widehat{\mathbf{U}}_{r}^{H}(\omega) \widehat{\mathbf{g}}_{o}\left(\mathbf{y}^{s}, \omega\right)\right] \widehat{\mathbf{U}}_{r}(\omega)
$$

for each frequency in the support of the probing pulse $\widehat{f}(\omega)$.

Our simulations assume that $\lambda \leq \ell<<a=(N-1) \lambda / 2<<L$, where $\lambda$ is the central wavelength, $\ell$ is a characteristic length scale of the inhomogeneity (like a correlation length), $a$ is the array aperture, and $L$ is the approximate distance to the targets from the array. This is the regime where multipathing, or multiple scattering, is significant even when the standard deviation of sound speed fluctuations is only a few percent.

We solve the wave equation in $2 \mathrm{D}$ with a numerical method based on the discretization of the mixed velocity-pressure formulation for acoustics. For the spatial discretization we use a new finite element method (Bécache et al., 2000), which is compatible with mass-lumping techniques (i.e., it leads to explicit time 
discretization schemes) and for the time discretization we use a centered second order finite difference scheme. In the numerical simulations, we have statistically homogeneous Gaussian random velocity fields generated using a random Fourier series, with constant mean $c_{0}=1.5 \mathrm{~km} / \mathrm{s}$, and exponential correlation function having correlation length $\ell=0.3 \mathrm{~mm}$ and standard deviation ranging from $1 \%$ to $5 \%$. The probing pulse is given by (4).

$$
f(t)=-2 \pi^{2} \nu^{2}\left(t-\frac{1}{\nu}\right) e^{-\pi^{2} \nu^{2}\left(t-\frac{1}{\nu}\right)^{2}}
$$

The central frequency is $\nu=3 \mathrm{MHz}$ and $\widehat{f}(\omega),(\omega=2 \pi \nu)$ is supported over the band of frequencies $0.159-7.958 \mathrm{MHz}$. The carrier wavelength is $\lambda=0.5 \mathrm{~mm}$ and the aperture of the array is $a=2.5 \mathrm{~mm}$. Then, the targets, which are soft scatterers, are modeled by small squares. The size of a small target is $\lambda / 30 \times \lambda / 30$, while the size of a larger one is $\lambda / 15 \times \lambda / 15$. Simulations are done on individual realizations, to be consistent with what happens in practice; there is no averaging of results from many realizations here. Furthermore, the same realization is typically used for the tests of all the imaging methods. Whenever the parameters ( $s$ and M.F.) are fixed, the realization is also fixed; but when the parameters change, the realization necessarily has changed. More details concerning the simulations may be found in (Bécache et al., 2000; Borcea et al., 2002).

We purposely present all the formulas in their most general form in terms of Green's functions. Thus, these formulas are valid either in 2D, as is relevant specifically to our simulations, or in 3D. The comparisons shown here use the Hankel-function fields in free space for the 2D simulations, but these functions would be replaced by point source Green's functions of the form $e^{i k r} / 4 \pi r$ for realistic data applications in 3D. Due to the high cost of numerically simulating wave propagation in a random medium, with significant multipathing, we only did 2D simulations up to now.

\section{Frequency domain methods: not statistically stable}

Two well-known frequency-domain imaging methods, matched-field processing (Bucker, 1976; Baggeroer et al., 1988; Baggeroer et al., 1993; Jensen et al., 1994; D’Spain et al., 1999) and MUSIC (Schmidt, 1979; Johnson, 1982; Johnson and DeGraaf, 1982; Schmidt, 1986; Stoica and Nehoral, 1989; Stoica and Moses, 1997; Devaney, 2002), will be presented next to show how these methods behave in the types of random media we are considering.

\subsection{Matched field - central frequency}

For matched-field processing, we compute

$$
\mathcal{G}_{\mathrm{MF}}^{(j)}\left(\mathbf{y}^{s}\right)=\left|\widehat{\mathbf{U}}_{j}^{H}(\omega) \widehat{\mathbf{g}}_{0}\left(\mathbf{y}^{s}, \omega\right)\right|^{2},
$$

and display the objective functional

$$
\mathcal{R}_{\mathrm{MF}}\left(\mathbf{y}^{s}\right)=\sum_{j=1}^{M} \frac{\mathcal{G}_{\mathrm{MF}}^{(j)}\left(\mathbf{y}^{s}\right)}{\max _{\mathbf{y}^{s}} \mathcal{G}_{\mathrm{MF}}^{(j)}\left(\mathbf{y}^{s}\right)}
$$

for a discrete set of points (usually regularly spaced on a grid which then aids in the ultimate computer display) $\mathbf{y}^{s}$ in the target domain.

Examples of matched-field processing with one or two targets are displayed in Figs. 1 and 2. 
The standard matched field (SMF) processing (Jensen et al., 1994) uses a somewhat different functional operating directly on $\widehat{P}(\omega) \widehat{P}^{H}(\omega)$. In our notation, the Bartlett-type objective functional of (Jensen et al., 1994) is

$$
\mathcal{R}_{\mathrm{SMF}}\left(\mathbf{y}^{s}\right)=\sum_{j=1}^{M} \sigma_{j}^{2}(\omega) \mathcal{G}_{\mathrm{SMF}}^{(j)}\left(\mathbf{y}^{s}\right),
$$

where $\sigma_{j}^{2}(\omega)$ is the singular value corresponding to the singular vector $\widehat{\mathbf{U}}_{j}(\omega)$ and

$$
\mathcal{G}_{\mathrm{SMF}}^{(j)}\left(\mathbf{y}^{s}\right)=\frac{\left|\widehat{\mathbf{U}}_{j}^{H}(\omega) \widehat{\mathbf{g}}_{0}\left(\mathbf{y}^{s}, \omega\right)\right|^{2}}{\left|\widehat{\mathbf{g}}_{0}\left(\mathbf{y}^{s}, \omega\right)\right|^{2}} .
$$

If there is a small number of targets and these targets do not differ significantly in strength at the central frequency (as is the case in our simulations), then there is no essential difference between the two imaging functionals. If, for example, we want to identify only the strongest scatterer, then we may consider only the first term in either sum, since the singular vectors are ordered according to their corresponding singular values from largest to smallest.

\subsection{MUSIC - central frequency}

For the MUSIC algorithm, we compute

$$
\mathcal{G}_{\operatorname{MUSIC}}\left(\mathbf{y}^{s}\right)=\left|\mathcal{P}_{N} \widehat{\mathbf{g}}_{0}\left(\mathbf{y}^{s}, \omega\right)\right|^{2},
$$

with $\mathcal{P}_{N} \widehat{\mathbf{g}}_{0}\left(\mathbf{y}^{s}, \omega\right)$ defined by (3). We display the objective functional

$$
\mathcal{R}_{\operatorname{MUSIC}}\left(\mathbf{y}^{s}\right)=\frac{\min _{\mathbf{y}^{s}} \mathcal{G}_{\text {MUSIC }}\left(\mathbf{y}^{s}\right)}{\mathcal{G}_{\operatorname{MUSIC}}\left(\mathbf{y}^{s}\right)},
$$

for points $\mathbf{y}^{s}$ in the target domain.

Examples for MUSIC with one or two targets in homogeneous and random media are displayed in Figs. 3 and 4.

When there is only one target in a homogeneous medium, then there is only one significant eigenvector of the array response matrix and it is proportional to $\widehat{\mathbf{g}}_{0}\left(\mathbf{y}^{t}, \omega\right)$, where $\mathbf{y}^{t}$ is the target location. It is clear in this case that both the MF and the MUSIC functionals have only a single term which peaks when $\mathbf{y}^{s}=\mathbf{y}^{t}$. Similarly, in the case of several targets in a homogeneous medium, the significant eigenvectors are linear combinations of $\widehat{\mathbf{g}}_{0}\left(\mathbf{y}_{j}^{t}, \omega\right)$, where $\mathbf{y}_{j}^{t}$ are the target locations and the functionals will have local maxima at the target locations.

In both of these frequency domain examples, it is clear from the Figures that no range information is obtained from these objective functionals in random media, and even the cross-range information is often quite haphazard. Indeed, as the random fluctuations in the velocity increase, there are false peaks and the functionals may not peak at the targets at all. (Note that MUSIC does provide the correct range for the zero variance case.) Lack of statistical stability prevents these imaging approaches from being useful in random media with significant multipathing considered here. When the realization of the random medium is changed, the images obtained typically change also - which is what we mean by the phrase "lack of statistical stability" for these methods.

\section{Statistically stable time domain methods}

Next we consider the same two methods of the previous section, but now we transform back to the timedomain to take advantage of the statistical stability that can be gained this way. 


\subsection{Matched field in time}

For matched-field processing, we compute

$$
\mathcal{G}_{\mathrm{MF}_{T}}^{(j)}\left(\mathbf{y}^{s}, t\right)=\int e^{-i \omega t} \sigma_{j}(\omega)\left|\widehat{\mathbf{U}}_{j}^{H}(\omega) \widehat{\mathbf{g}}_{0}\left(\mathbf{y}^{s}, \omega\right)\right|^{2} d \omega
$$

Since the factor multiplying $e^{-i \omega t}$ in the integrand is real and nonnegative, this integral clearly takes its maximum value for $t=0-$ which is also, therefore, the location of the peak of the corresponding pulse in the time domain. We then display the objective functional

$$
\mathcal{R}_{\mathrm{MF}_{T}}\left(\mathbf{y}^{s}\right)=\sum_{j=1}^{M} \frac{\mathcal{G}_{\mathbf{M F}^{s}}^{(j)}\left(\mathbf{y}^{s}, t=0\right)}{\max _{\mathrm{MF}_{T}}\left(\mathbf{y}^{s}, t=0\right)},
$$

for points $\mathbf{y}^{s}$ in the target domain.

Examples of matched-field processing in the time domain with one or two targets are displayed in Figs. 5 and 6.

\subsection{DOA (time domain MUSIC)}

The next method is a time domain variant of MUSIC which we will label $D O A$, because it gives very good estimates of the direction of arrival. (Note that matched field in time also gives good estimates of the direction of arrival).

To define a time variant of MUSIC, we first normalize the projection $\mathcal{P}_{N} \widehat{\mathbf{g}}_{0}\left(\mathbf{y}^{s}, \omega\right)$ (given by (3)) by the singular value $\sigma_{j}(\omega)$ and then take the inverse Fourier transform to go to the time domain,

$$
\begin{aligned}
& \mathcal{F}^{(j)}\left(\mathbf{y}^{s}, t\right)=\int e^{-i \omega t} \sigma_{j}(\omega) \widehat{\mathbf{g}}_{o}\left(\mathbf{y}^{s}, \omega\right) d \omega \\
& -\int e^{-i \omega t} \sigma_{j}(\omega) \sum_{r=1}^{M}\left[\widehat{\mathbf{U}}_{r}^{H}(\omega) \widehat{\mathbf{g}}_{o}\left(\mathbf{y}^{s}, \omega\right)\right] \widehat{\mathbf{U}}_{r}(\omega) d \omega .
\end{aligned}
$$

Theoretical analysis shows that the functionals $\mathcal{F}^{(j)}\left(\mathbf{y}^{s}, t\right)$ are statistically stable, i.e they behave like deterministic quantities, and thus it seems natural to evaluate them at deterministic times (i.e., simple geometrical spreading times). We then form the sum

$$
\mathcal{G}^{(j)}\left(\mathbf{y}^{s}\right)=\sum_{p=1}^{N}\left|\mathcal{F}_{p}^{(j)}\left(\mathbf{y}^{s}, t_{p}\left(\mathbf{y}^{s}\right)\right)\right|^{2},
$$

and display the objective functional

$$
\mathcal{R}_{\mathrm{DOA}}\left(\mathbf{y}^{s}\right)=\sum_{j=1}^{M} \frac{\min _{\mathbf{y}^{s}} \mathcal{G}^{(j)}\left(\mathbf{y}^{s}\right)}{\mathcal{G}^{(j)}\left(\mathbf{y}^{s}\right)},
$$

for points $\mathbf{y}^{s}$ in the target domain.

The arrival time $t_{p}\left(\mathbf{y}^{s}\right)$ is the deterministic travel time from the $p$-th transducer to the search point,

$$
t_{p}\left(\mathbf{y}^{s}\right)=\frac{\left|\mathbf{x}_{p}-\mathbf{y}^{s}\right|}{c_{0}}
$$

Examples for time-domain MUSIC with one or two targets are displayed in Figs. 7 and 8.

The cross-range results are now dramatically improved in both methods. Range information is still not to be found here, but the statistical stability of the universal "comet tails" is now easily observed. The images shown are for specific realizations, but the results do not change significantly when the underlying realization of the random medium is changed. This fact has been repeatedly shown in our simulations, and is the main characteristic of statistically stable methods. 


\section{Time domain processing and range estimation methods}

To complete the localization of the targets, we also need an estimate of the range. Good range estimates can be obtained in the near field either from amplitude moveout information or from arrival time information. In the far field, only the arrival time information is useful, and we will concentrate on arrival times in the present analysis.

One commonly used range estimator is Synthetic Aperture Imaging or $S A I$. Another alternative that arises in the time-reversal approach is the use of arrival time information in the singular vectors. This arrival time information can also be averaged for random media - see (Borcea et al., 2002) - to obtain very stable estimates of arrival times. Both of these methods will now be combined with the time-domain methods of the previous section to obtain well-localized images of the targets.

\subsection{Matched field in time combined with SAI}

The SAI objective function is

$$
\mathcal{R}_{\mathrm{SAI}}\left(\mathbf{y}^{s}\right)=\sum_{p=1}^{N} P_{p p}\left(2 t_{p}\left(\mathbf{y}^{s}\right)\right) .
$$

At a search point $\mathbf{y}^{s}$ in the domain of interest, we compute the deterministic arrival time for diagonal entry $P_{p p}(t)$. That is, the time to go from the $p$-th transducer to the search point $\mathbf{y}^{s}$ and then come back to the $p$-th transducer. This is twice the arrival time $t_{p}\left(\mathbf{y}^{s}\right)$ given by (16). Note that the SAI functional gives good range information even in random media (see (Borcea et al., 2002)).

The idea here is to combine the functional $S A I$ which give good range information with the functional $M F_{T}$ which provide good cross range resolution in order to get good images. One way of doing this combination is to display

$$
\mathcal{R}_{\mathrm{MF}_{T}-\mathrm{SAI}}\left(\mathbf{y}^{s}\right)=\sum_{j=1}^{M} \frac{\mathcal{G}_{\mathrm{MF}_{T}-\mathrm{SAI}}^{(j)}\left(\mathbf{y}^{s}\right)}{\max _{\mathbf{y}^{s}} \mathcal{G}_{\mathrm{MF}_{T}-\mathrm{SAI}}^{(j)}\left(\mathbf{y}^{s}\right)}
$$

where

$$
\mathcal{G}_{\mathrm{MF}_{T} \text {-SAI }}^{(j)}\left(\mathbf{y}^{s}\right)=\left|\sum_{p=1}^{N} P_{p p}\left(2 t_{p}\left(\mathbf{y}^{s}\right)\right)\right| \mathcal{G}_{\mathrm{MF}_{T}}^{(j)}\left(\mathbf{y}^{s}, t=0\right) .
$$

Examples of time-domain matched-field processing with Synthetic Aperture Imaging as the range estimator for one or two targets are displayed in Figs. 9 and 10. The method is statistically stable and gives good estimates of the target locations.

\subsection{Matched field in time combined with times from averaged singular vectors}

We would like to use the singular vectors $\widehat{\mathbf{U}}_{j}(\omega)$ to estimate the travel times from target $j$ to the array. Remark though that the singular vectors $\widehat{\mathbf{U}}_{j}(\omega)$ which are normalized $\left(\left\|\widehat{\mathbf{U}}_{j}(\omega)\right\|=1\right)$ carry an arbitrary, frequency dependent, phase. Because of this $\mathbf{U}_{j}(t)$ look incoherent in the time domain. We can, however, calculate $N$, coherent in time, versions of singular vectors by projecting the columns of the response matrix onto them

$$
\widehat{\mathbf{U}}_{j}^{(p)}(\omega)=\left[\widehat{\mathbf{U}}_{j}(\omega)^{H} \widehat{\mathbf{P}}^{(p)}(\omega)\right] \widehat{\mathbf{U}}_{j}(\omega), p=1, \ldots, N, j=1, \ldots, M
$$


Here $\widehat{\mathbf{P}}^{(p)}$ is the $p^{\text {th }}$ column of the response matrix $\widehat{P}(\omega)$. Clearly $\widehat{\mathbf{U}}_{j}^{(p)}(\omega)$ are singular vectors of $\widehat{P}(\omega)$ and carry the phase of its $p^{\text {th }}$ column. We use these various versions of the singular vectors to estimate $\tau_{p}^{(j)}$, for $j=1, \ldots, M$, and $p=1, \ldots, N$, the travel times from target $j$ to the array element $p$ as the minimizers of

$$
\min _{\tau_{p}^{(j)}} \int_{0}^{T} \sum_{p=1}^{N}\left|\mathbf{U}_{j}^{(p)}\left(t-\tau_{p}^{(j)}\right)-\frac{1}{N} \sum_{q=1}^{N} \mathbf{U}_{j}^{(q)}\left(t-\tau_{q}^{(j)}\right)\right|^{2} d t .
$$

The ATSV (Arrival Times from averaged Singular Vectors) functional is defined by

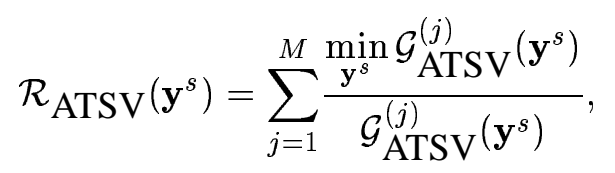

where

$$
\mathcal{G}_{\mathrm{ATSV}}^{(j)}\left(\mathbf{y}^{s}\right)=\sum_{p=1}^{N}\left[\tau_{p}^{(j)}-2 t_{p}\left(\mathbf{y}^{s}\right)\right]^{2} .
$$

We combine $M F_{T}$ with $A T S V$ to obtain

$$
\mathcal{R}_{\operatorname{MF}_{T}-\operatorname{ATSV}}\left(\mathbf{y}^{s}\right)=\sum_{j=1}^{M} \frac{\mathcal{G}_{\mathrm{MF}_{T}-\operatorname{ATSV}}^{(j)}\left(\mathbf{y}^{s}\right)}{\max _{\mathbf{y}^{s}} \mathcal{G}_{\mathrm{MF}_{T^{-}}-\operatorname{ATSV}}\left(\mathbf{y}^{s}\right)},
$$

where

$$
\mathcal{G}_{\operatorname{MF}_{T}-\operatorname{ATSV}}^{(j)}\left(\mathbf{y}^{s}\right)=\mathcal{G}_{\mathrm{MF}_{T}}^{(j)}\left(\mathbf{y}^{s}\right) / \mathcal{G}_{\mathrm{ATSV}}^{(j)}\left(\mathbf{y}^{s}\right) .
$$

Examples of time-domain matched-field processing with arrival-time estimates from the averaged singular vectors for one or two targets are displayed in Figs. 11 and 12. This method is also statistically stable and gives good estimates of the target locations. The results look somewhat better than those in Figs. 9 and 10 , and considerably better for stronger fluctuations.

\subsection{DOA combined with SAI}

The DOA-SAI estimator is

$$
\mathcal{R}_{\text {DOA-SAI }}\left(\mathbf{y}^{s}\right)=\sum_{j=1}^{M} \frac{\mathcal{G}_{\text {DOA-SAI }}^{(j)}\left(\mathbf{y}^{s}\right)}{\max _{\mathbf{y}^{s}} \mathcal{G}_{\text {DOA-SAI }}^{(j)}\left(\mathbf{y}^{s}\right)}
$$

where

$$
\mathcal{G}_{\text {DOA-SAI }}^{(j)}\left(\mathbf{y}^{s}\right)=\left|\sum_{p=1}^{N} P_{p p}\left(2 t_{p}\left(\mathbf{y}^{s}\right)\right)\right| / \mathcal{G}^{(j)}\left(\mathbf{y}^{s}\right),
$$

and $\mathcal{G}^{(j)}\left(\mathbf{y}^{s}\right)$ is given by (14).

Examples of time-domain MUSIC with Synthetic Aperture Imaging estimates as range estimator for one or two targets are displayed in Figs. 13 and 14. This method is also statistically stable and gives good estimates of the target locations. 


\subsection{DOA combined with arrival times from averaged singular vectors}

For each search point $\mathbf{y}_{s}$, we compute the objective functional

$$
\mathcal{R}_{\mathrm{SAT}}\left(\mathbf{y}^{s}\right)=\sum_{j=1}^{M} \frac{\min _{\mathbf{y}^{s}} \mathcal{G}_{\mathrm{SAT}^{(j)}}^{\left(\mathbf{y}^{s}\right)}}{\mathcal{G}_{\mathrm{SAT}^{(j)}}^{\left(\mathbf{y}^{s}\right)}}
$$

where

$$
\mathcal{G}_{\mathrm{SAT}}^{(j)}\left(\mathbf{y}^{s}\right)=\sum_{p=1}^{N} \mid \mathcal{F}_{p}^{(j)}\left(\mathbf{y}^{s},\left.t_{p}\left(\mathbf{y}^{s}\right)\right|^{2}\left[\tau_{p}^{(j)}-t_{p}\left(\mathbf{y}^{s}\right)\right]^{2} .\right.
$$

Here $\mathcal{F}^{(j)}\left(\mathbf{y}^{s}, t\right)$ is defined by (13), $t_{p}\left(\mathbf{y}^{s}\right)$, for $p=1, \ldots, N$, are the deterministic arrival times given by (16) and $\tau_{p}^{(j)}$, for $p=1, \ldots, N$, and $j=1, \ldots, M$, are the arrival times computed in (21). We call (28) the Subspace Arrival Time (SAT) estimator.

Examples of SAT or time-domain MUSIC with arrival time estimates from the averaged singular vectors for one or two targets are displayed in Figs. 15 and 16. This method is again statistically stable and gives good estimates of the target locations. The results again look somewhat better than those in Figs. 13 and 14. These localization results have apparently degraded the least of all those considered here at the highest values of the random fluctuations.

\section{CONCLUSIONS}

For imaging applications in randomly inhomogeneous acoustical media, the foregoing results lead us to the following conclusions: (1) Single frequency methods (including MUSIC and D.O.R.T.) are not statistically stable, and therefore cannot be used without modification in the presence of significant amounts of spatial heterogeneity in the acoustic wave speed distribution. (2) In contrast, time domain methods are statistically stable for any objective functional having the characteristic that the random Green's functions appear in Hermitian conjugate pairs of $g g^{*}$ (Borcea et al., 2002). This result has been shown here to be true for DOA and Matched Field, and is expected to be true more generally. (Of course, statistical stability is a necessary, but not a sufficient, condition for optimal imaging in random media, so satisfaction of this criterion is not enough in itself.) (3) The DOA estimates seem superior to those of Matched Field, but a careful analysis shows that this is only superficially so because of the quantity chosen for display in these plots. In the homogeneous case, exact calculations show that this superficial difference is just related to shapes (not the locations) of the peaks in each of the objective functionals, and therefore is only a display issue and not fundamental. (4) Both DOA and Matched Field give only cross-range or bearing information. Range information must be obtained separately.

To locate the targets in random media, we need either multiple views (using multiple arrays) so we can triangulate, or we need to extract a direct measure of range from the data. Both arrival time and amplitude moveout (i.e., changes in peak arrival time and amplitude correlated directly with the array element locations) contain sufficient information to extract range. Usefulness of amplitudes is limited by the range itself; if the range is so large that the arrivals appear as planar across the array aperture then only the plane-wave arrival time is useful. In the examples chosen here, we concentrated on arrival time and this information was obtained by combining MF with either SAI or ATSV (arrival times from averaged singular vectors). As anticipated, results obtained after intersection with SAI are not as good as the ones obtained after intersection with ATSV. But the results were shown nevertheless to be a good compromise because this method does not require a separate arrival time analysis (recall that SAI is not statistically stable (Borcea et al., 2002)). 
Our main conclusion is that there is no essential difference in imaging with MF or DOA when either SAI or ATSV are used.

\section{Acknowledgments}

We thank W. A. Kuperman and H. C. Song for helpful leads into the array processing literature. We thank D. H. Chambers for useful comments on the manuscript. The work of J. G. Berryman was performed under the auspices of the U.S. Department of Energy by the University of California Lawrence Livermore National Laboratory under contract No. W-7405-ENG-48 and supported specifically by the LLNL Laboratory Directed Research and Development Program and Environmental Management Sciences Program. The work of L. Borcea was partially supported by the National Science Foundation under grant numbers DMS-9971209 and DMS97-09320, by DOE DE-FG03-00ER25424, and by ONR grant N00014-02-1-0088. The work of G. C. Papanicolaou was supported by grants AFOSR F49620-01-1-0465, NSF DMS-9971972, DOE DE-FG03-00ER25424, and ONR N00014-02-1-0088.
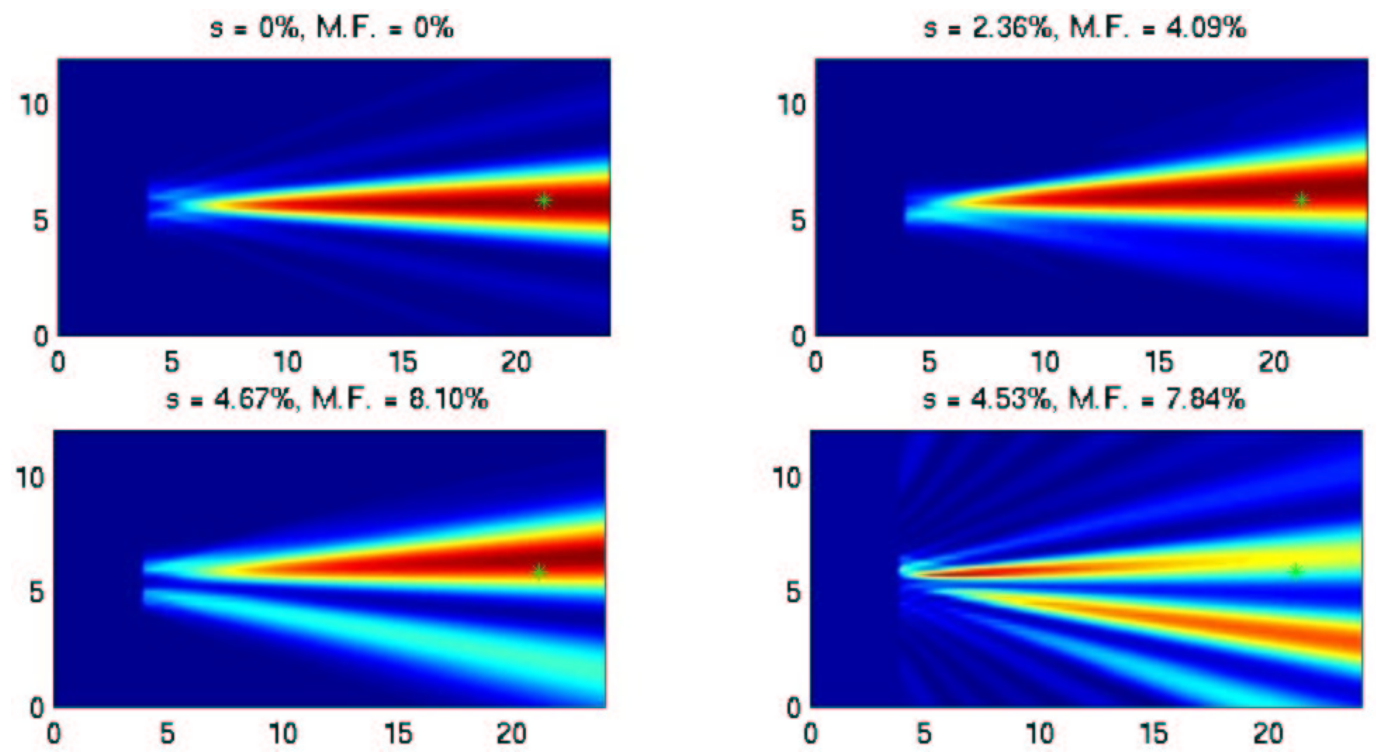

Figure 1: The Matched Field central frequency [ $M F$ estimate (6)] estimate of the location of one target in random media with different strength of the fluctuations of the sound speed. The exact location of the target is denoted by the green star. The standard deviation $s$ and maximum fluctuations (M.F.) are indicated on the top of each view. The horizontal axis is the range in $\mathrm{mm}$ and the vertical axis is the cross-range in $\mathrm{mm}$. 

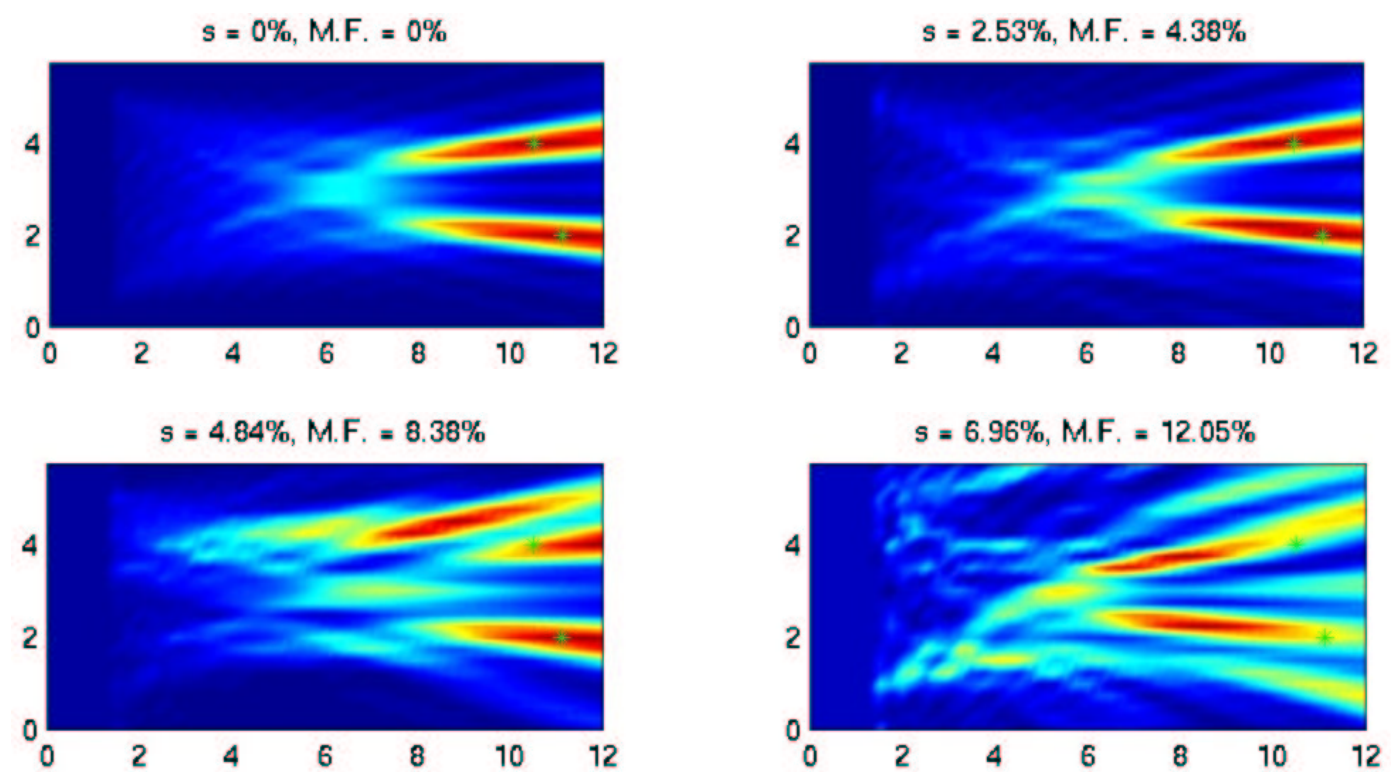

Figure 2: Similar to Fig. 1 but with two targets.
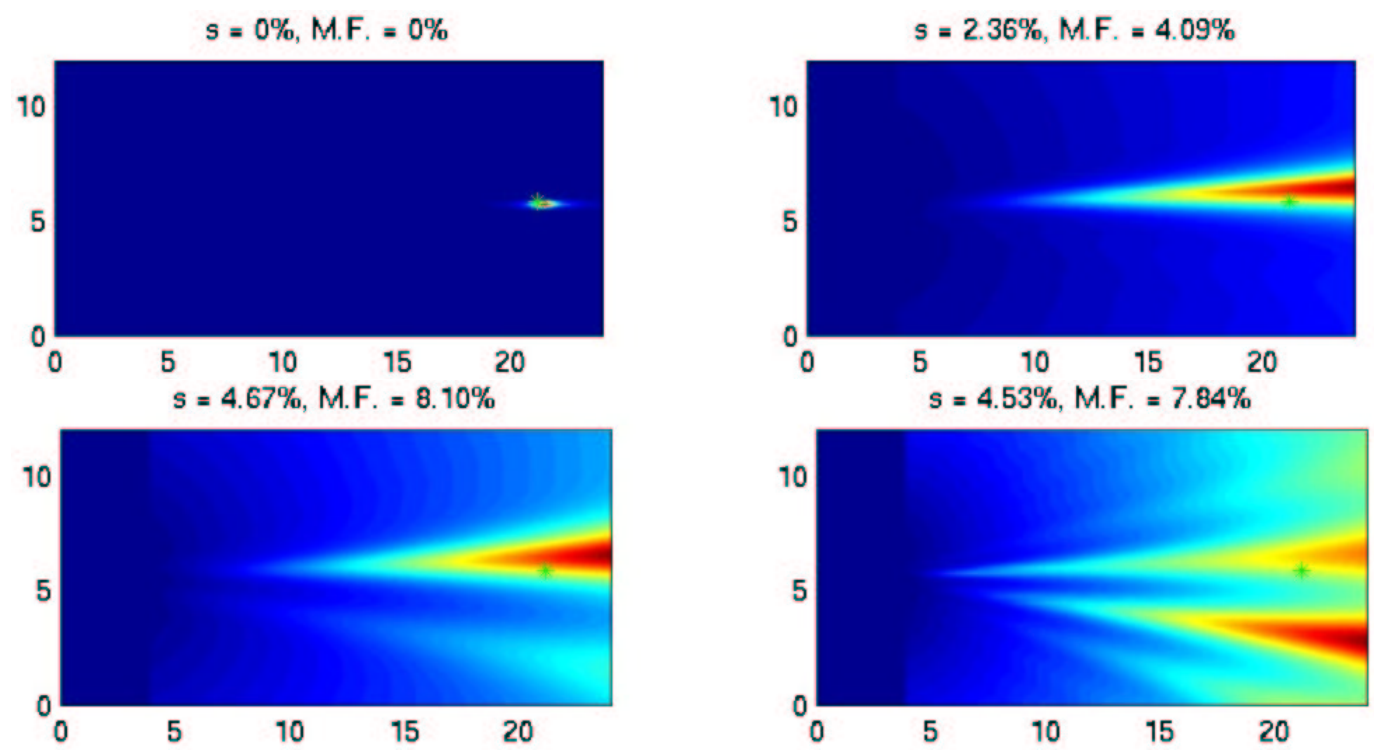

Figure 3: The MUSIC central frequency estimate [MUSIC estimate (10)] of the location of one target in random media with different strength of the fluctuations of the sound speed. The exact location of the target is denoted by the green star. The standard deviation $s$ and maximum fluctuations (M.F.) are indicated on the top of each view. The horizontal axis is the range in $\mathrm{mm}$ and the vertical axis is the cross-range in mm. 

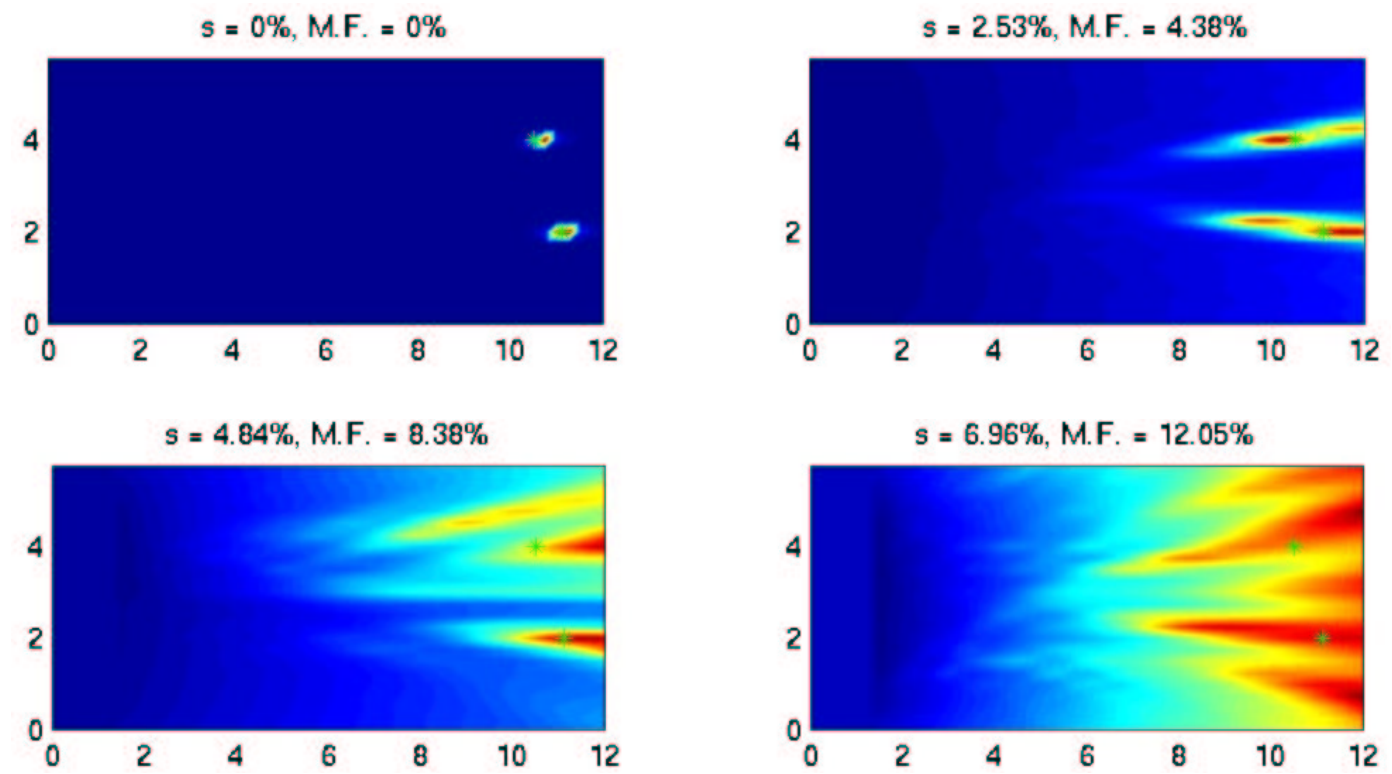

Figure 4: Similar to Fig. 3 but with two targets.
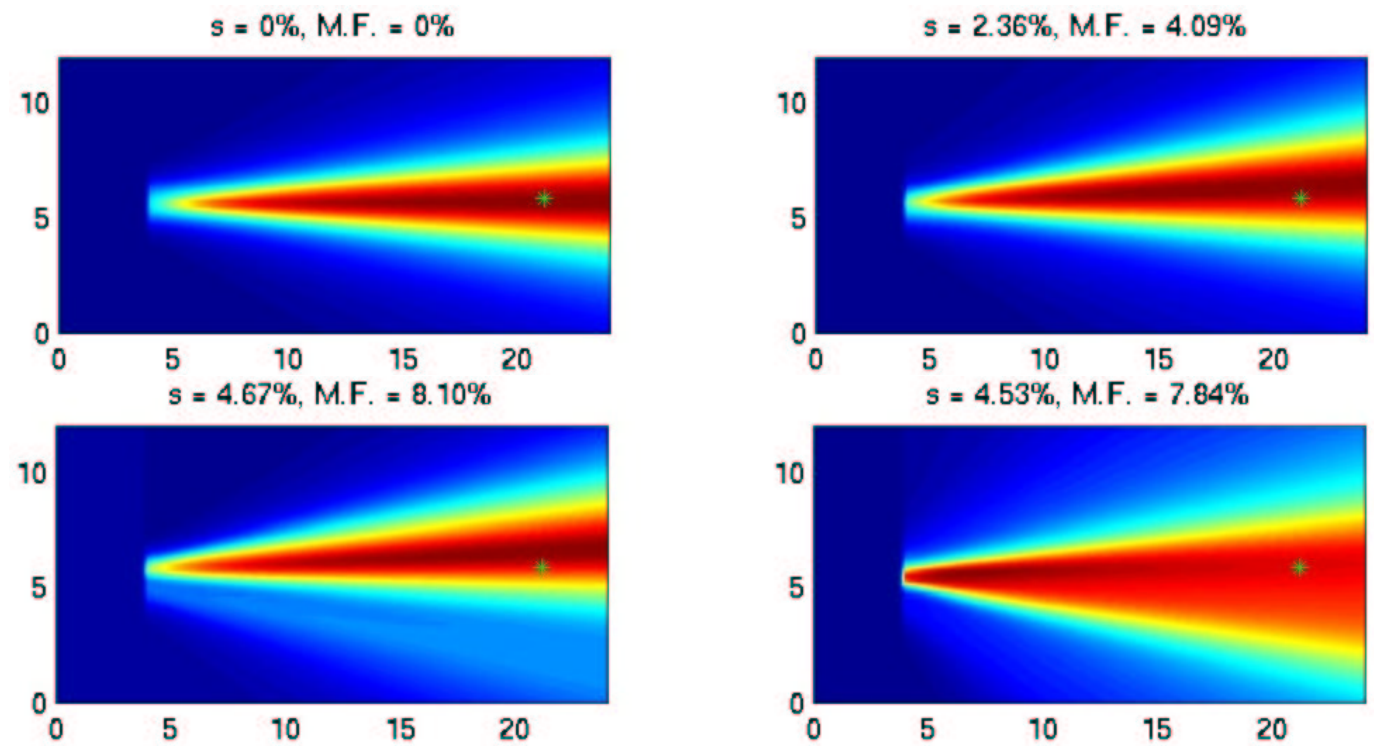

Figure 5: The Matched Field time estimate $\left[M F_{T}\right.$ estimate (12)] of the location of one target in random media with different strength of the fluctuations of the sound speed. The exact location of the target is denoted by the green star. The standard deviation $s$ and maximum fluctuations (M.F.) are indicated on the top of each view. The horizontal axis is the range in $\mathrm{mm}$ and the vertical axis is the cross-range in $\mathrm{mm}$. 

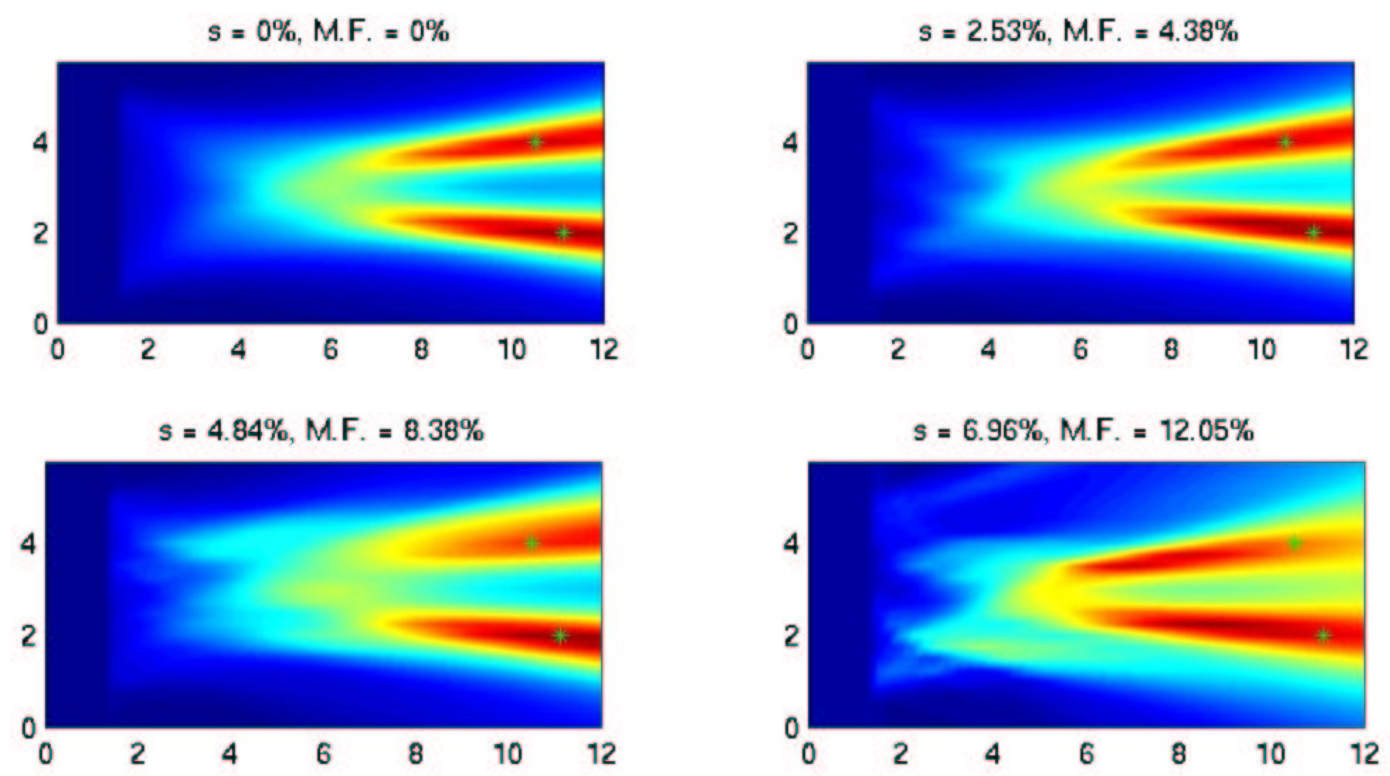

Figure 6: Similar to Fig. 5 but with two targets.
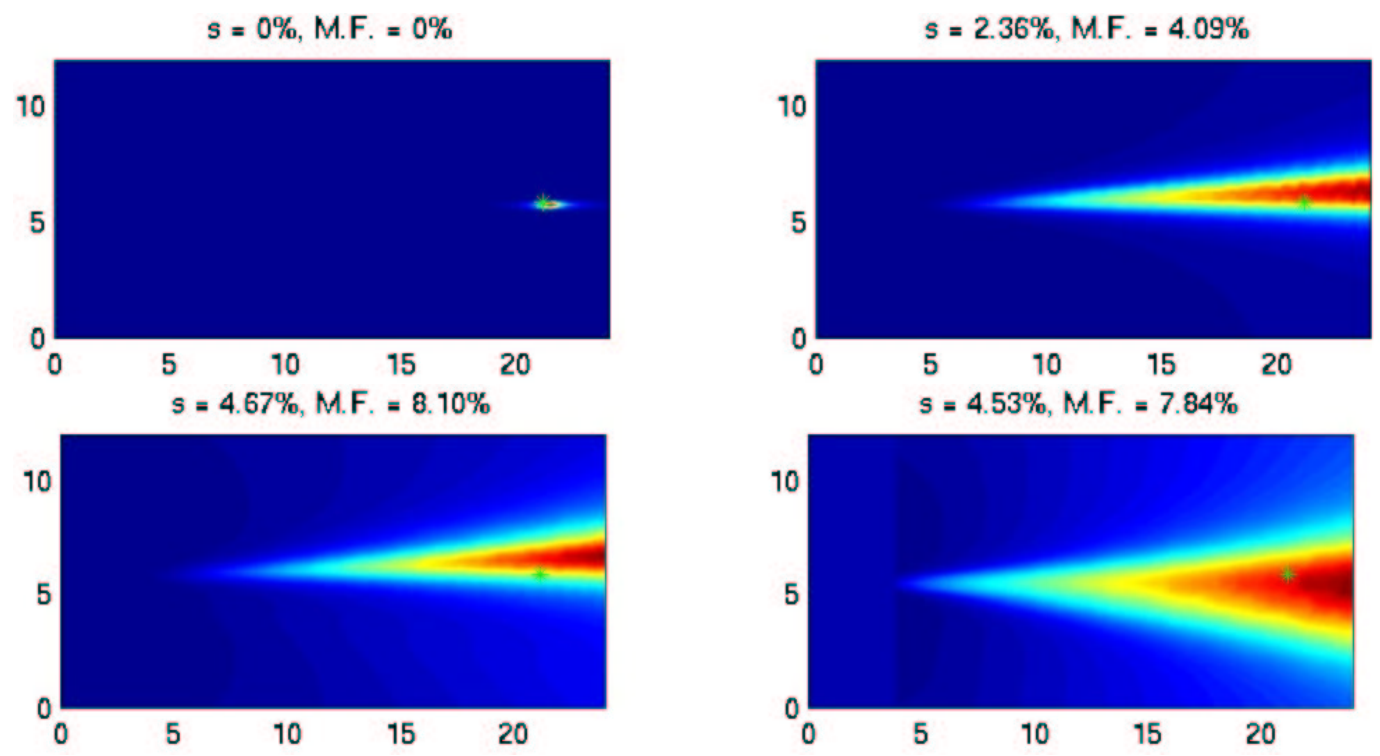

Figure 7: The DOA estimate [DOA estimate (15)] of the location of one target in random media with different strength of the fluctuations of the sound speed. The exact location of the target is denoted by the green star. The standard deviation $s$ and maximum fluctuations (M.F.) are indicated on the top of each view. The horizontal axis is the range in $\mathrm{mm}$ and the vertical axis is the cross-range in $\mathrm{mm}$. 

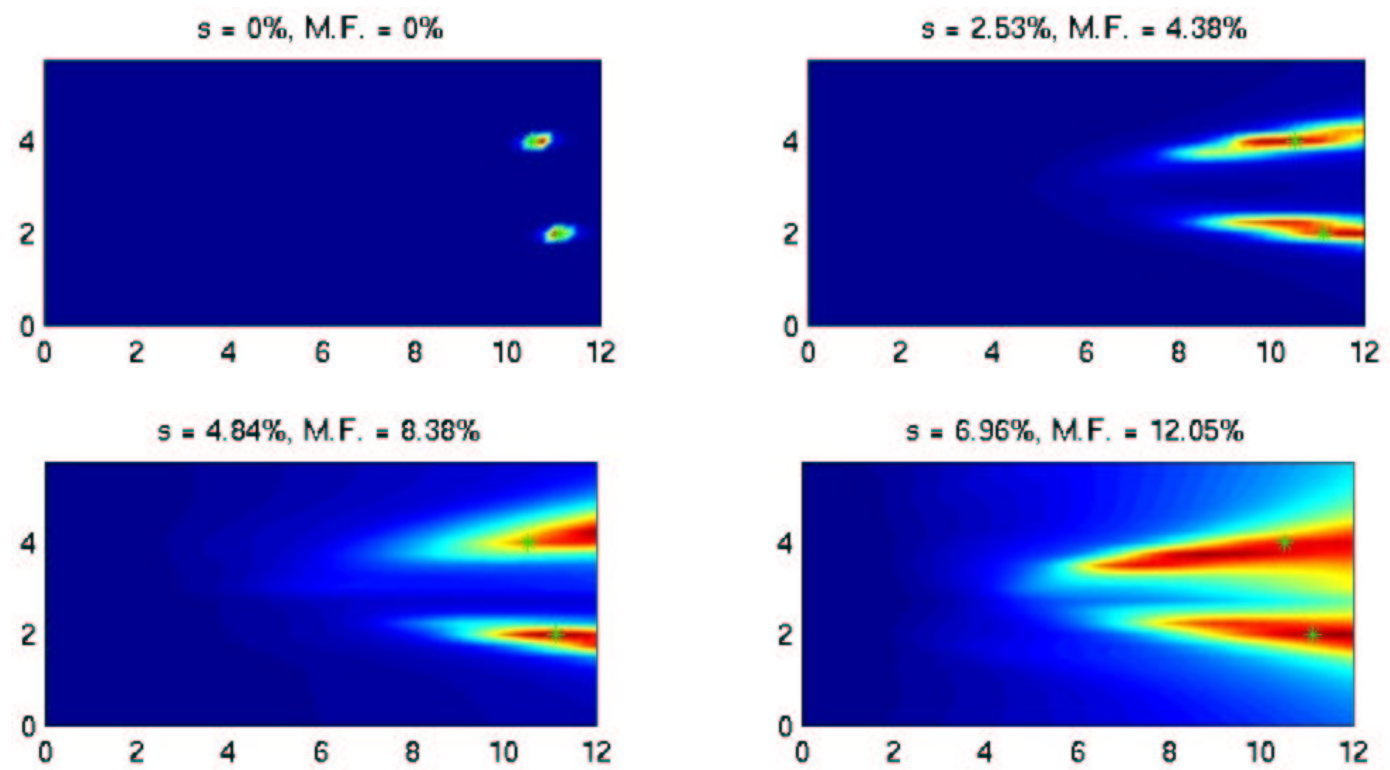

Figure 8: Similar to Fig. 7 but with two targets.
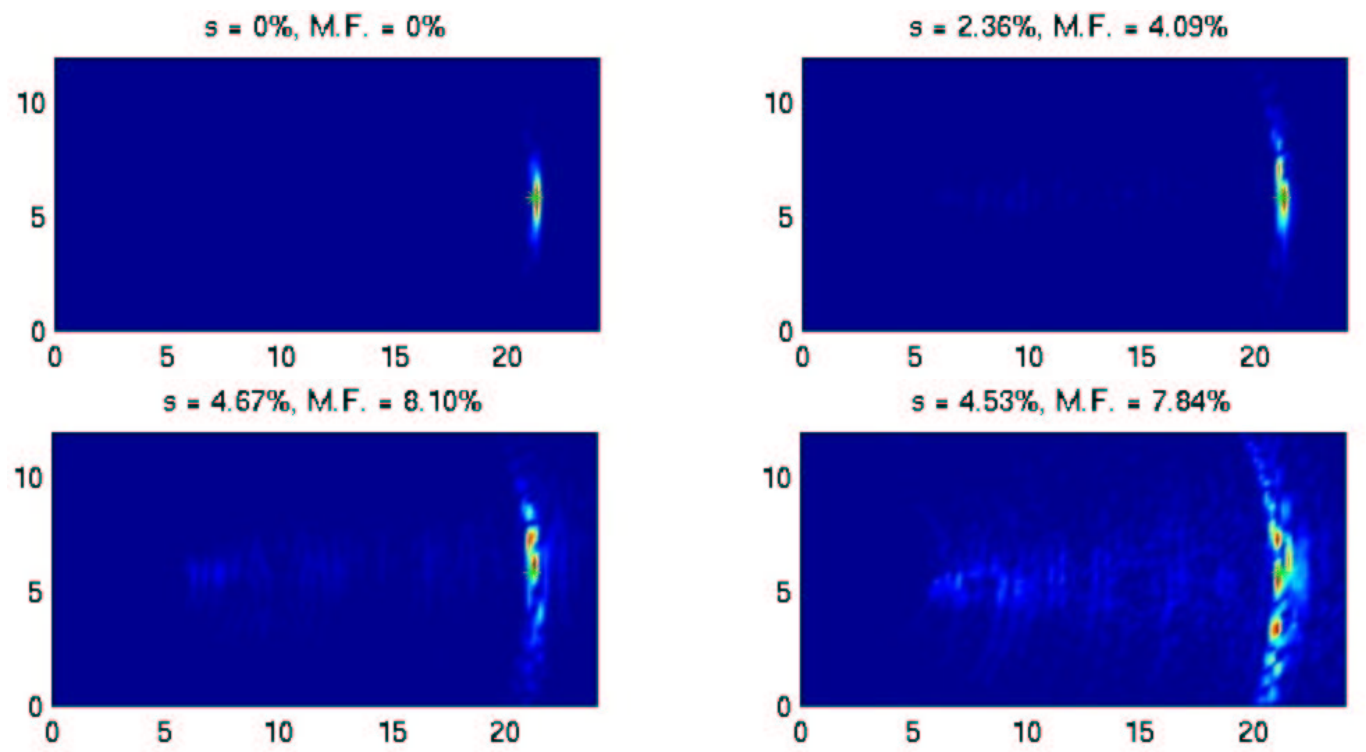

Figure 9: Combined $M F_{T}$ and $S A I$ [ $M F_{T^{-}} S A I$ estimate (18)] estimation of the location of one target in random media with different strength of the fluctuations of the sound speed. The exact location of the target is denoted by the green star. The standard deviation $s$ and maximum fluctuations (M.F.) are indicated on the top of each view. The horizontal axis is the range in $\mathrm{mm}$ and the vertical axis is the cross-range in mm. 

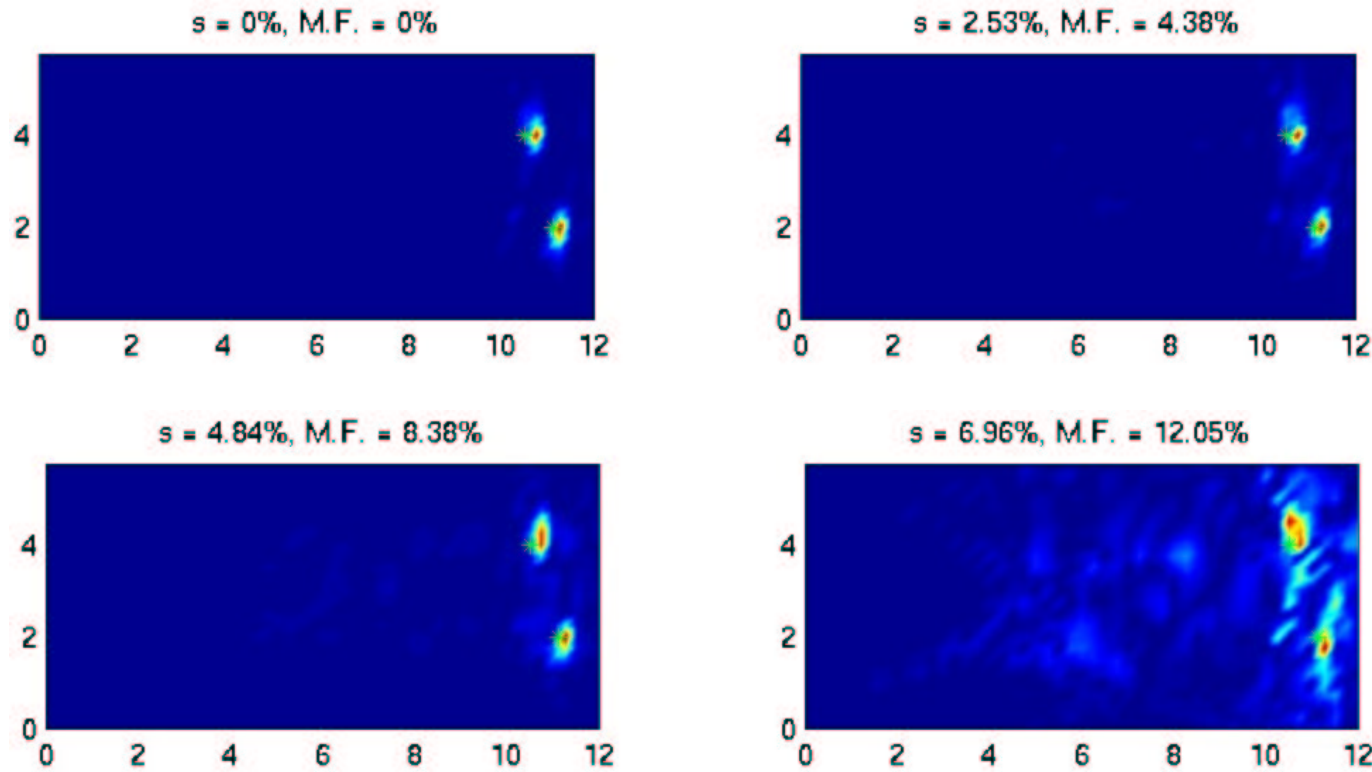

Figure 10: Similar to Fig. 9 but with two targets.
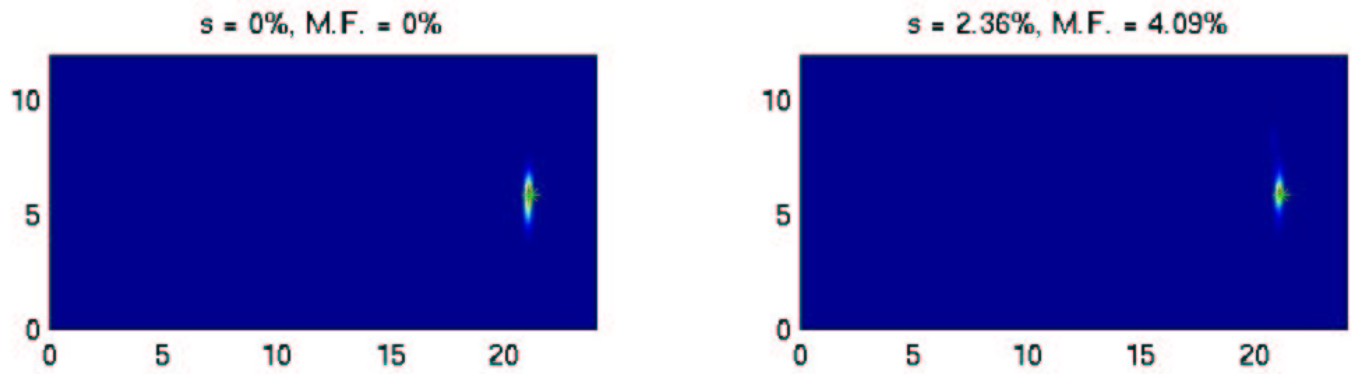

$s=4.67 \%, M . F .=8.10 \%$
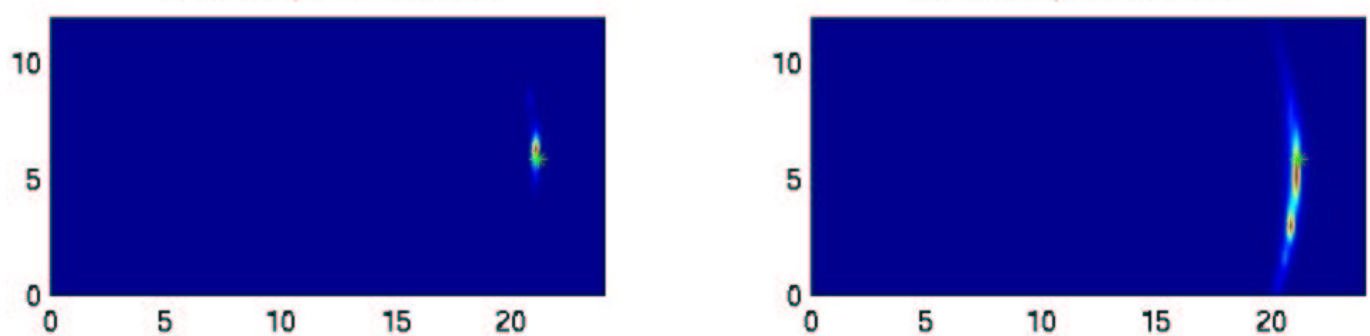

Figure 11: Combined $M F_{T}$ and $A T S V$ [ $M F_{T}-A T S V$ estimate (24)] estimation of one target location in random media with different strength of the fluctuations of the sound speed. The exact location of the target is denoted by the green star. The standard deviation $s$ and maximum fluctuations (M.F.) are indicated on the top of each view. The horizontal axis is the range in $\mathrm{mm}$ and the vertical axis is the cross-range in $\mathrm{mm}$. 

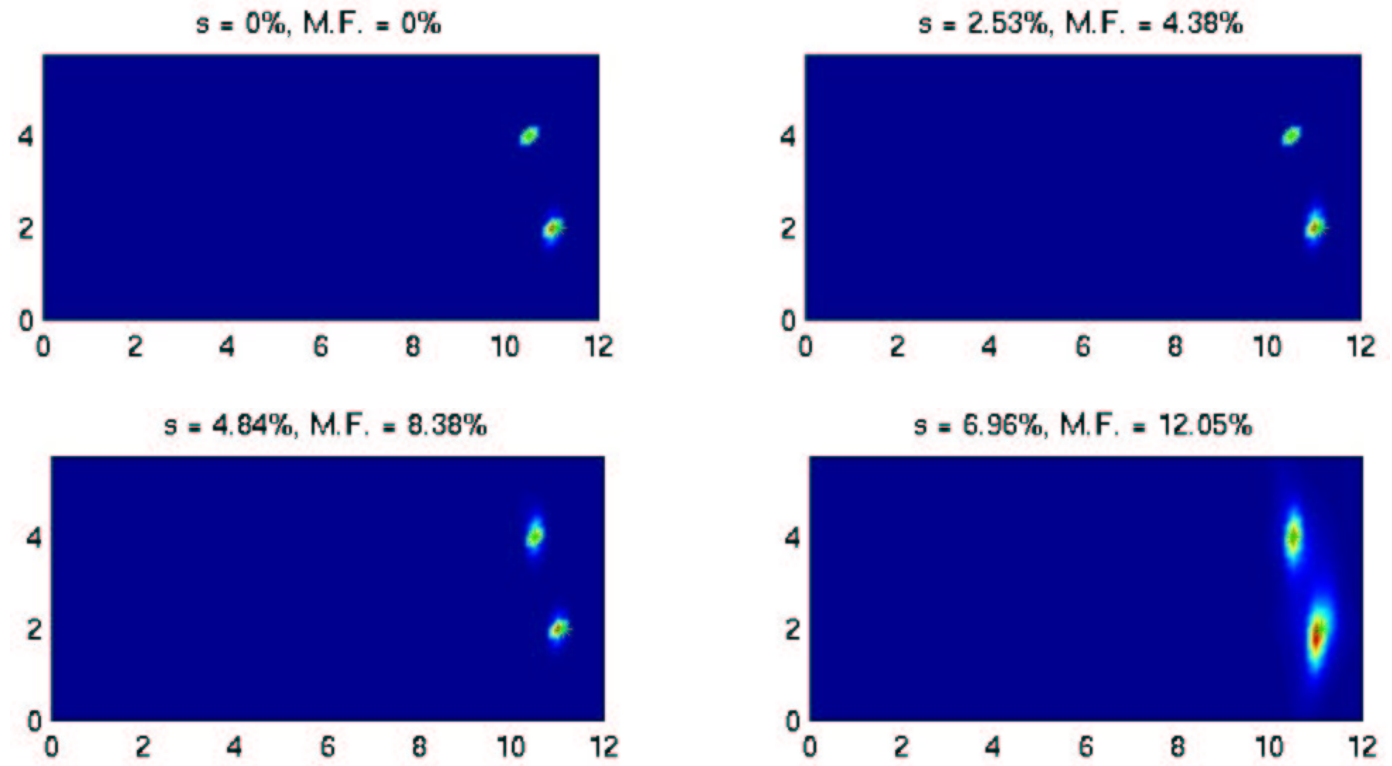

Figure 12: Similar to Fig. 11 but with two targets.
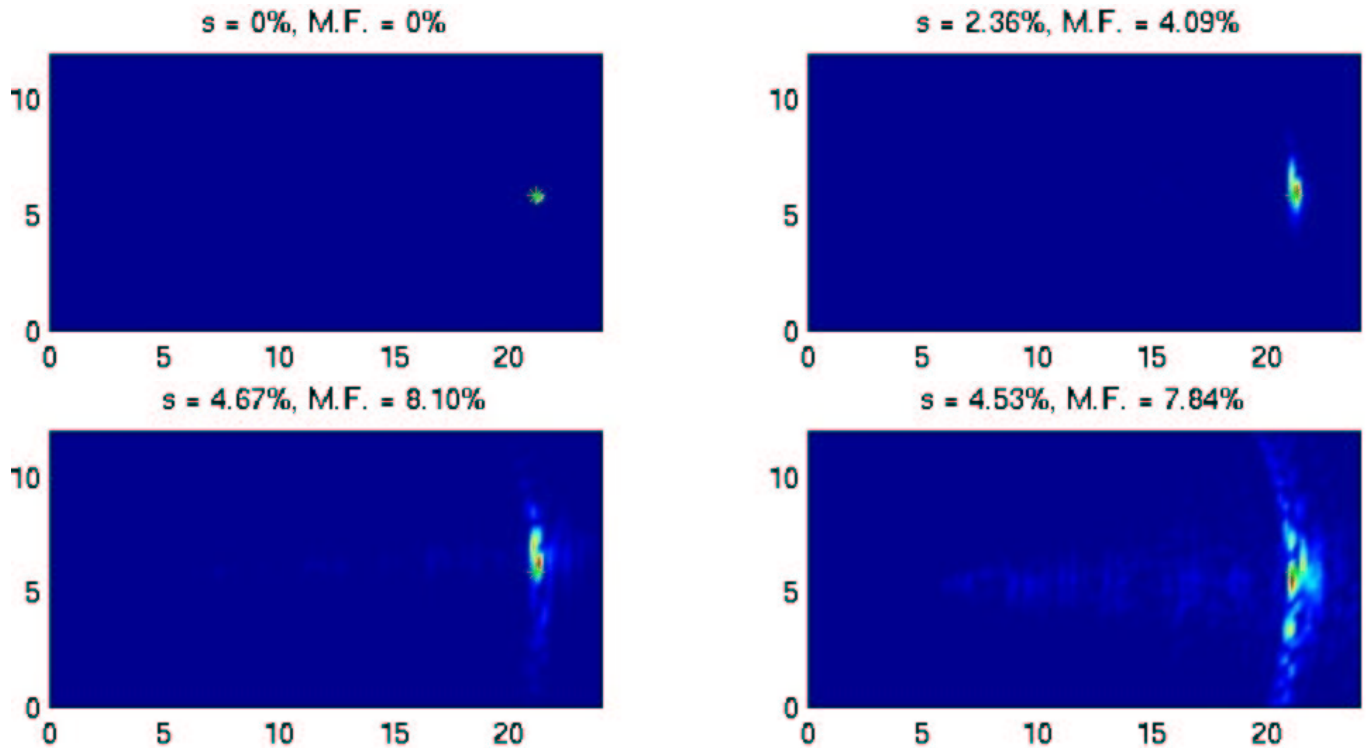

Figure 13: Combined DOA and SAI [ $D O A-S A I$ estimate (26)] estimation of one target location in random media with different strength of the fluctuations of the sound speed. The exact location of the target is denoted by the green star. The standard deviation $s$ and maximum fluctuations (M.F.) are indicated on the top of each view. The horizontal axis is the range in $\mathrm{mm}$ and the vertical axis is the cross-range in mm. 

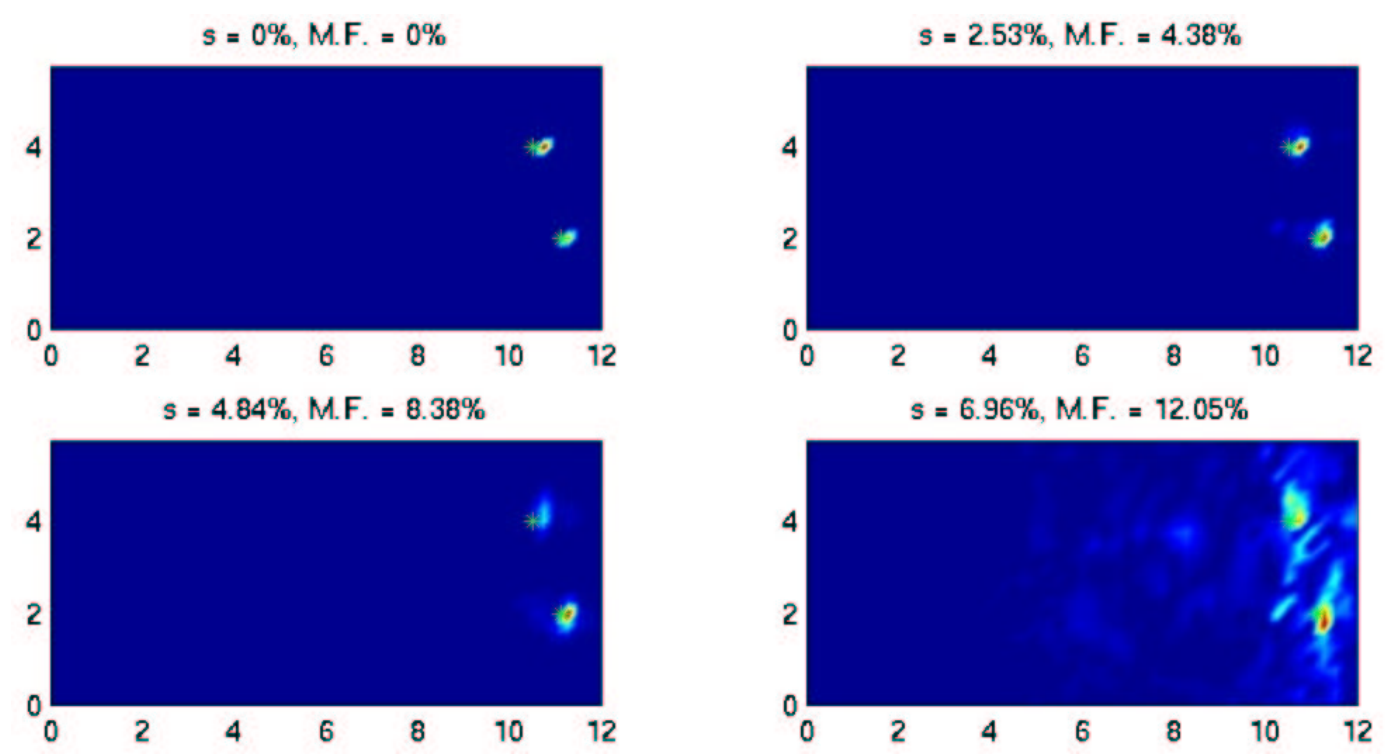

Figure 14: Same as Fig. 13 with two targets.
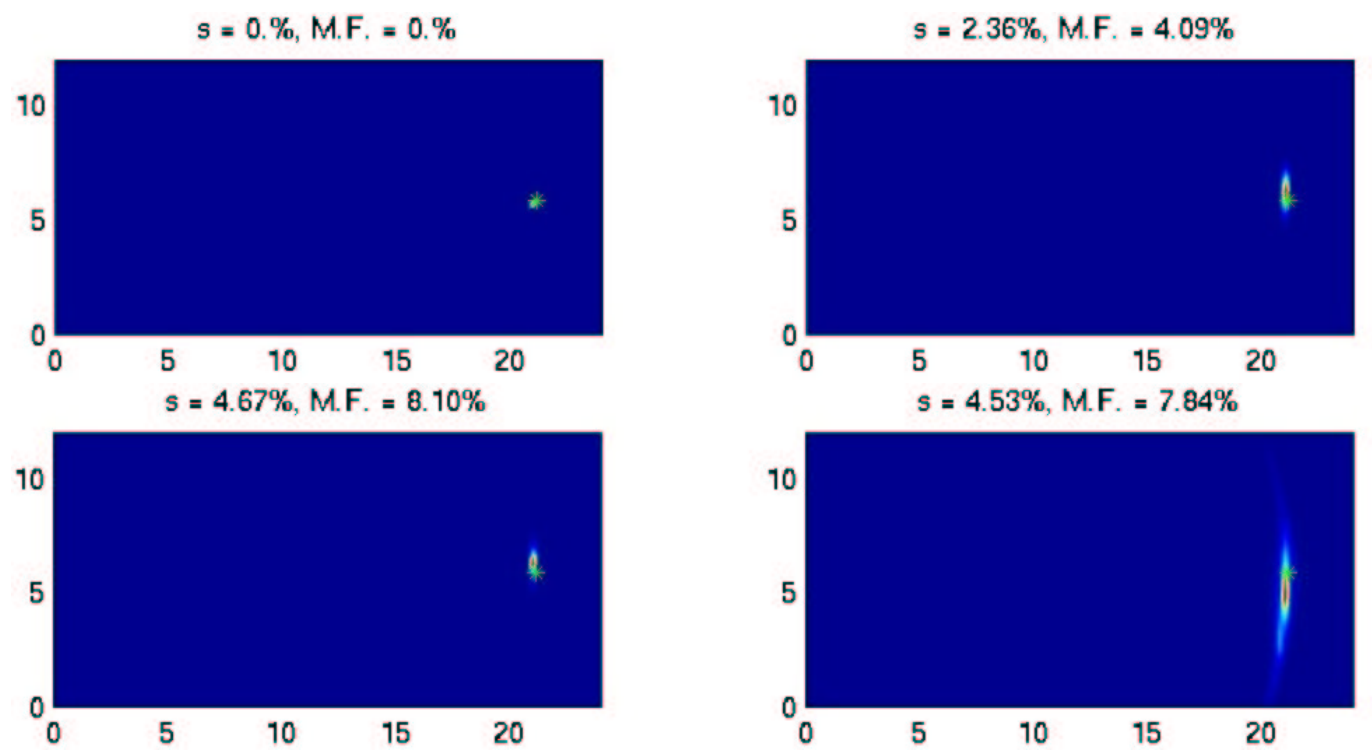

Figure 15: The SAT estimate [estimate (28)] of one target in random media with different strength of the fluctuations of the sound speed. The exact location of the target is denoted by the green star. The standard deviation $s$ and maximum fluctuations (M.F.) are indicated on the top of each view. The horizontal axis is the range in $\mathrm{mm}$ and the vertical axis is the cross-range in $\mathrm{mm}$. 

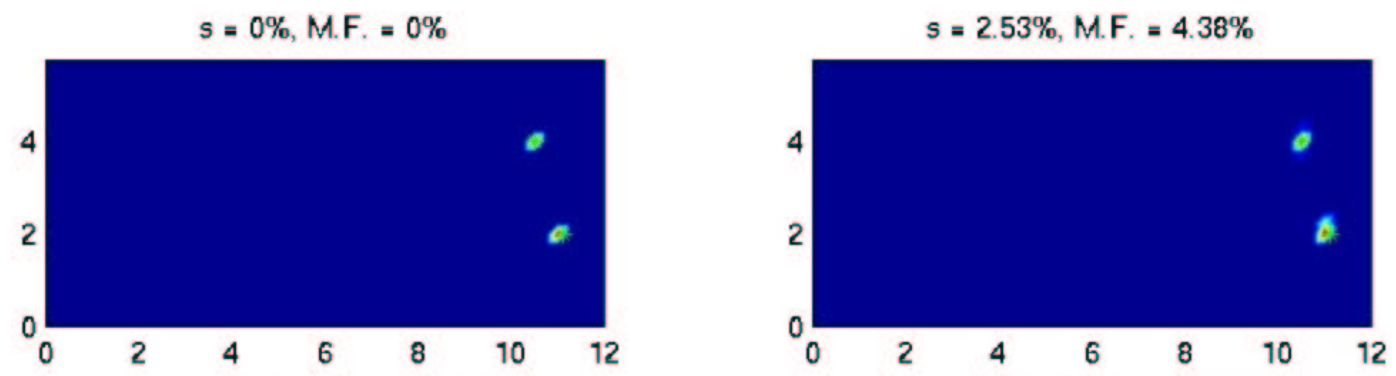

$s=4.84 \%, M . F .=8.38 \%$

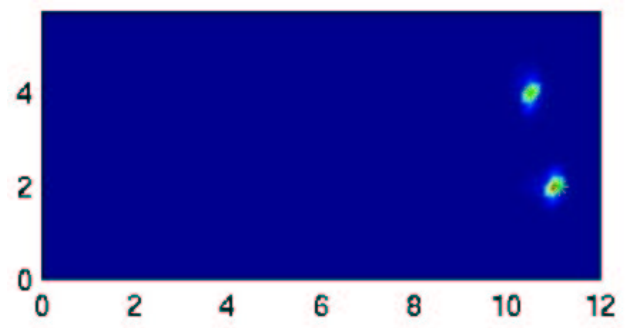

$s=6.96 \%$, M.F. $=12.05 \%$

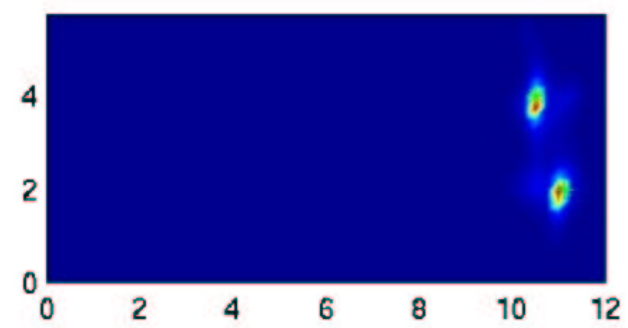

Figure 16: The SAT estimate as in Fig. 15 for two targets. 


\section{References}

Aki, K. and Richards, P. G. (1980). Quantitative Seismology: Theory and Methods, volume II. Freeman, New York.

Baggeroer, A. B., Kuperman, W. A., and Mikhalevsky, P. N. (1993). An overview of matched field methods in ocean acoustics. IEEE J. Oceanic Engng., 18:401-424.

Baggeroer, A. B., Kuperman, W. A., and Schmidt, H. (1988). Matched field processing: Source localization in correlated noise as an optimum parameter estimation problem. J. Acoust. Soc. Am., 83:571-587.

Bécache, E., Joly, P., and Tsogka, C. (2000). An analysis of new mixed finite elements for the approximation of wave propagation problems. SIAM J. Numer. Anal., 37:1053-1084.

Bleistein, N., Cohen, J. K., and Stockwell, Jr., J. W. (2001). Mathematics of Multidimensional Seismic Imaging, Migration, and Inversion. Springer, New York.

Blomgren, P., Papanicolaou, G., and Zhao, H. (2002). Super-resolution in time-reversal acoustics. J. Acoust. Soc. Am., 111:238-248.

Borcea, L., Papanicolaou, G. C., Tsogka, C., and Berryman, J. G. (2002). Imaging and time reversal in random media. currently under review for publication in Inverse Problems.

Bucker, H. P. (1976). Use of calculated sound field and matched-field detection to locate sound sources in shallow water. J. Acoust. Soc. Am., 59:368-373.

Burridge, R., Papanicolaou, G., Sheng, P., and White, B. (1989). Probing a random medium with a pulse. SIAM J. Appl. Math., 49:582-607.

Burridge, R., Papanicolaou, G., and White, B. (1986). Statistics of reflected pulses. Lecture Notes in Mathematics, 1186:265-270.

Burridge, R., Papanicolaou, G., and White, B. (1987). Statistics for pulse reflection from a randomly layered medium. SIAM J. Appl. Math., 47:146-168.

Burridge, R., Papanicolaou, G., and White, B. (1988). One-dimensional wave-propagation in a highly discontinuous medium. Wave Motion, 10:19-44.

Cheney, M. (2001). The linear sampling method and the music algorithm. Inverse Problems, 17:591-596.

Cheney, M., Isaacson, D., and Newell, J. C. (1999). Electrical impedance tomography. SIAM Rev., 41:85101.

Claerbout, J. F. (1976). Fundamentals of Geophysical Data Processing with Applications to Petroleum Prospecting. McGraw-Hill, New York.

Clouet, J. F. and Fouque, J.-P. (1997). A time reversal method for an acoustical pulse propagating in randomly layered media. Wave Motion, 25:361-368.

Devaney, A. J. (2002). Super-resolution processing of multi-static data using time reversal and MUSIC. to appear in JASA (2002).

D’Spain, G. L., Murray, J. J., Hodgkiss, W. S., Booth, N. O., and Schey, P. W. (1999). Mirages in shallow water matched field processing. J. Acoust. Soc. Am., 105:3245-3265. 
Fink, M. (2001). Chaos and time-reversed acoustics. Physica Scripta, T90:268-277.

Fink, M. (March, 1997). Time reversal acoustics. Physics Today, 50:34-40.

Fink, M. (November, 1999). Time-reversed acoustics. Scientific American, 281:91-97.

Fink, M., Cassereau, D., Derode, A., Prada, C., Roux, P., Tanter, M., Thomas, J.-L., and Wu, F. (2000). Time-reversed acoustics. Rep. Prog. Phys., 63:1933-1995.

Fink, M. and Prada, C. (2001). Acoustic time-reversal mirrors. Inverse Problems, 17:R1-R38.

Fink, M., Prada, C., and Wu, F. (1989). Self focusing in inhomogeneous media with time reversal acoustic mirrors. In McAvoy, B. R., editor, Proc. IEEE Ultrason. Symp. 1989, volume 2, pages 681-686.

Floch, C. L., Tanter, M., and Fink, M. (1999). Self-defocusing in ultrasonic hyperthermia: Experiment and simulation. Appl. Phys. Lett., 74:3062-3064.

Ishimaru, A. (1978). Wave Propagation and Scattering in Random Media. Academic Press, New York.

Jackson, D. R. and Dowling, D. R. (1991). Phase conjugation in underwater acoustics. J. Acoust. Soc. Am., 89:171-181.

Jensen, F. B., Kuperman, W. A., Porter, M. B., and Schmidt, H. (1994). Computational Ocean Acoustics. AIP Press, New York.

Johnson, D. H. (1982). The application of spectral estimation methods to bearing estimation problems. Proc. IEEE, 70:1018-1028.

Johnson, D. H. and DeGraaf, S. R. (1982). Improving the resolution of bearing in passive sonar arrays by eigenvalue analysis. IEEE Trans. Acoustics, Speech, Signal Proc., ASSP-30:638-647.

Karal Jr., F. C. and Keller, J. B. (1964). Elastic, electromagnetic, and other waves in a random medium. $J$. Math. Phys., 5:537-547.

Keller, J. B. (1962). Wave propagation in random media. In 13th Symposium in Applied Mathematics, New York, 1960, pages 227-246, Providence, Rhode Island. American Mathematical Society.

Keller, J. B. (1964). Stochastic equations and wave propagation in random media. In 16th Symposium in Applied Mathematics, New York, 1963, pages 145-170, Providence, Rhode Island. American Mathematical Society.

Keller, J. B. and Karal Jr., F. C. (1966). Effective dielecric constant, permeability, and conductivity of a random medium and the velocity and attenuation coefficient of coherent waves. J. Math. Phys., 7:661670.

Krolik, J. L. (1992). Matched-field minimum variance beamforming in a random ocean channel. J. Acoust. Soc. Am., 92:1408-1419.

Kuperman, W. A., Hodgkiss, W. S., Song, H. C., Akal, T., Ferla, C., and Jackson, D. R. (1998). Phase conjugation in the ocean: Experimental demonstration of an acoustic time-reversal mirror. J. Acoust. Soc. Am., 103:25-40.

Mast, T. D., Nachman, A. I., and Waag, R. C. (1997). Focusing and imaging using eigenfunctions of the scattering operator. J. Acoust. Soc. Am., 102:715-725. 
Mordant, N., Prada, C., and Fink, M. (1999). Highly resolved detection and selective focusing in a waveguide using the d.o.r.t. method. J. Acoust. Soc. Am., 105:2634-2642.

O’Doherty, R. F. and Anstey, N. A. (1971). Reflections on amplitudes. Geophys. Prospecting, 19:430-458.

Prada, C. and Fink, M. (1994). Eigenmodes of the time reversal operator: A solution to selective focusing in multiple-target media. Wave Motion, 20:151-163.

Prada, C., Manneville, S., Spoliansky, D., and Fink, M. (1996). Decomposition of the time reversal operator: Detection and selective focusing on two scatterers. J. Acoust. Soc. Am., 99:2067-2076.

Prada, C., Wu, F., and Fink, M. (1991). The iterative time reversal mirror: A solution to self-focusing in the pulse echo mode. J. Acoust. Soc. Am., 90:1119-1129.

Richards, P. G. and Menke, W. (1983). The apparent attenuation of a scattering medium. Bull. Seism. Soc. Am., 73:1005-1021.

Sabra, K. G., Khosla, S. R., and Dowling, D. R. (2002). Broadband time-reversing array retrofocusing in noisy environments. J. Acoust. Soc. Am., 111:823-830.

Schmidt, R. O. (1979). Multiple emitter location and signal parameter estimation. In Proc. RADC Spectrum Estimation Workshop, pages 243-258, Rome, NY. Rome Air Development Center. RADC-TR-79-63.

Schmidt, R. O. (1986). Multiple emitter location and signal parameter estimation. IEEE Trans. Antennas Propag., AP-34:276-281.

Solna, K. and Papanicolaou, G. (2000). Ray theory for a locally layered random medium. Waves Random Media, 10:151-198.

Steinberg, B. D. (1983). Microwave Imaging with Large Antenna Arrays. Wiley, New York.

Stoica, P. and Moses, R. (1997). Introduction to Spectral Analysis. Prentice Hall, New York.

Stoica, P. and Nehoral, A. (1989). Music, maximum likelihood, and cramer-rao bound. IEEE Trans. Acoustics, Speech, and Signal Processing, 17:720-741.

Tanter, M., Thomas, J.-L., and Fink, M. (1998). Influence of boundary conditions on time-reversal focusing through heterogeneous media. Appl. Phys. Lett., 72:2511-2513.

ter Haar, G. (December, 2001). Acoustic surgery. Physics Today, 54:29-34.

Thomas, J.-L. and Fink, M. A. (1996). Ultrasonic beam focusin through tissue inhomogeneities with a time reversal mirror: Application to transskull therapy. IEEE Trans. Ultrason. Ferroelectr. Freq. Control, 43:1122-1129. 